\title{
Article
}

http://dx.doi.org/10.11646/phytotaxa.175.4.4

\section{Studies on Wrightoporia from China 3. Wrightoporia subavellanea sp. nov. based on morphological characters and rDNA sequence data}

\author{
JIA-JIA CHEN \& BAO-KAI CUI* \\ Institute of Microbiology, PO Box 61, Beijing Forestry University, Beijing 100083, China \\ *Corresponding author's e-mail: baokaicui2013@gmail.com
}

\begin{abstract}
Wrightoporia subavellanea sp. nov. is described and illustrated from southern China on the basis of morphological characters and molecular data. It is characterized by an annual growth habit, resupinate basidiocarps with white rhizomorphs, large pores, narrow and strongly dextrinoid skeletal hyphae, broadly ellipsoid to subglobose, thick-walled, finely asperulate and strongly amyloid basidiospores (3.8-4.2 × 2.8-3.2 $\mu \mathrm{m})$, and presence of gloeoplerous hyphae in trama. A molecular study based the combined ITS (internal transcribed spacer region) and nLSU (the large nuclear ribosomal RNA subunit) dataset supported the position of the new species in Wrightoporia. A key to accepted species of Wrightoporia in China is provided.
\end{abstract}

Key words: Wrightoporiaceae, phylogeny, polypore, taxonomy, wood-inhabiting fungi

\section{Introduction}

Wrightoporia Pouzar (1966) was described and typified with W. lenta (Overh. \& J. Lowe) Pouzar. The principal characteristics of the genus include a combination of resupinate to pileate basidiocarps, an annual to perennial growth habit, a monomitic to trimitic hyphal structure, and amyloid asperulate basidiospores (Ryvarden 1982, David \& Rajchenberg 1987, Hattori 2008). About 39 species of Wrightoporia are accepted worldwide (Corner 1989, Teixeira 1992, Stalpers 1996, Lindblad \& Ryvarden 1999, Hattori 2003, 2008, Chen \& Cui 2012, Chen \& Yu 2012), of which 17 species have been recorded in China (Cui \& Dai 2006, Dai \& Cui 2006, Dai 2007, 2012, Dai \& Yuan 2007, Dai et al. 2011, Chen \& Cui 2012, Chen \& Yu 2012).

Investigations on the diversity of wood-inhabiting fungi in China have been carried out recently, and many new species were described based on morphological characters along with molecular evidence (Cui et al. 2011, He \& Dai 2012, Cui 2013, Cui \& Dai 2013, Cui \& Decock 2013, Li \& Cui 2013, Li et al. 2013, Zhao \& Cui 2013, Zhao et al. 2013). During a taxonomic study on Wrightoporia in China, one species new to science was found based on morphological characters and phylogenetic analysis of ITS and nLSU rDNA sequences, and its illustrated description was provided. In addition, an identification key to the accepted species of Wrightoporia in China was also provided.

\section{Materials and methods}

Morphological studies. - The studied specimens were deposited at the herbarium of the Institute of Microbiology, Beijing Forestry University (BJFC). The microscopic routine followed Dai et al. (2010). Sections were studied at magnification up to $\times 1000$ using a Nikon E80i microscope and phase contrast illumination. Drawings were made with the aid of a drawing tube. Microscopic features, measurements and drawings were made from slide preparations stained with Cotton Blue and Melzer's reagent. Spores were measured from sections cut from the tubes. Presenting the variation in the size of the spores, $5 \%$ of measurements were excluded from each end of the range, and were given in parentheses. Basidiospore spine lengths are not included in the measurements. In the text the following abbreviations were used: $\mathrm{KOH}=5 \%$ potassium hydroxide, $\mathrm{CB}=$ Cotton $\mathrm{Blue}, \mathrm{CB}+=$ cyanophilous, $\mathrm{L}=$ mean spore 
Wrightoporia and its related genera requires evolutionary information from wider taxa samplings and more conserved gene markers.

Until now, 18 species of Wrightoporia were found in China, among them, several species were failed to get the rDNA sequences data. In the present study, all the available sequences of Wrightoporia in China were included in the phylogenetic analysis, and an identification key to the accepted species of Wrightoporia in China was provided.

\section{Key to accepted species of Wrightoporia in China}

1. Hyphal system monomitic.... W. nigrolimitata Jia J. Chen

Hyphal system dimitic or trimitic . .2

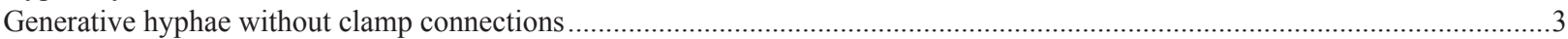

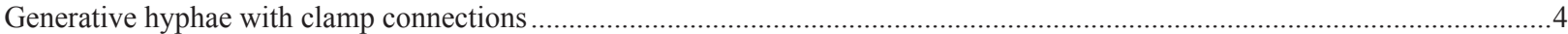

Basidiocarps without rhizomorphs ..................................................................................... casuarinicola Y.C. Dai \& B.K. Cui

Basidiocarps with rhizomorphs W. rubella Y.C. Dai

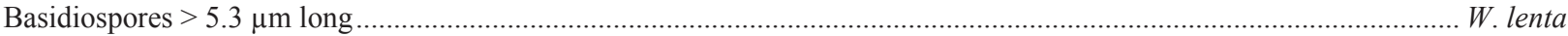

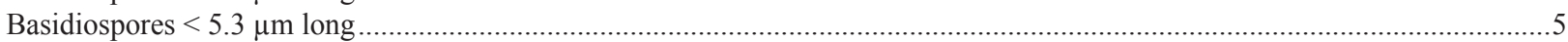

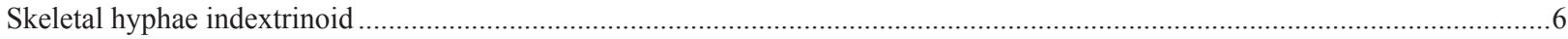

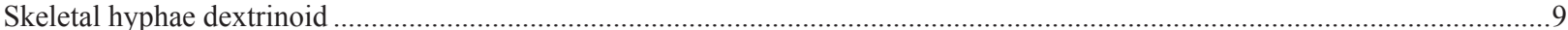

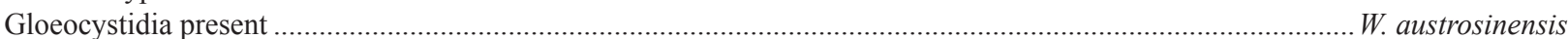

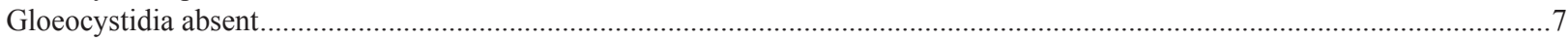

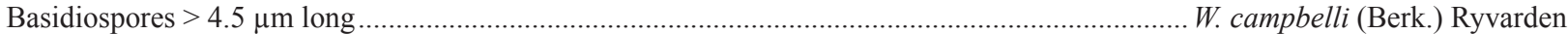

Basidiospores $<4.5 \mu \mathrm{m}$ long

Basidiospores $>2.6 \mu \mathrm{m}$ wide ............................................................................................................. W. cinnamomea Ryvarden

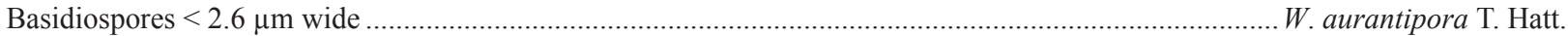

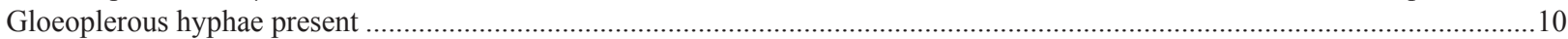

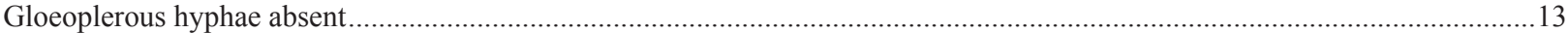

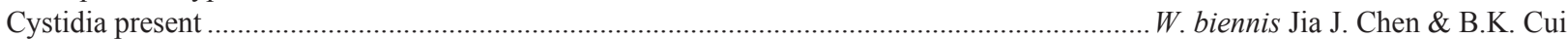

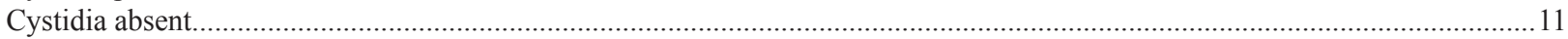

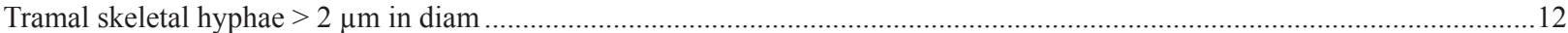

Tramal skeletal hyphae $<2 \mu \mathrm{m}$ in diam ........................................................................................................W. subavellanea

Pore 1-2 per mm; pore surface pale orange to greyish brown ...............................................................W. labyrinthina T. Hatt.

Pore 2-4 per mm; pore surface cream to buff-yellow ............................................................................... boreais Y.C.

Skeletal hyphae absent in the context..................................................W. ochrocrocea (Henn. \& E. Nyman) A. David \& Rajchenb.

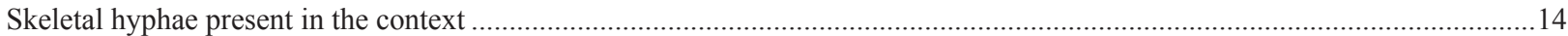

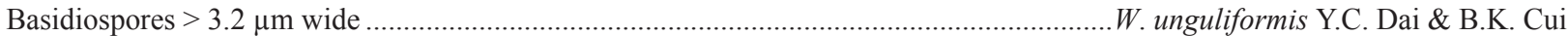

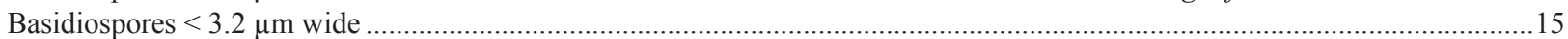

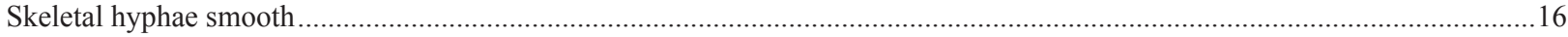

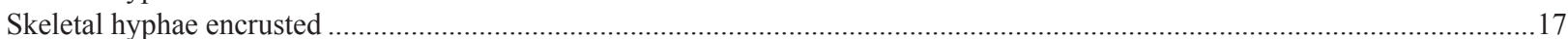

Basidiocarps annual; tramal skeletal hyphae $2-4 \mu \mathrm{m}$ in diam ................................................... . africana I. Johans. \& Ryvarden

Basidiocarps perennial; tramal skeletal hyphae 4-8 $\mu \mathrm{m}$ in diam .............................................................................. W. tropicalis

Generative hyphae with both clamp connections and simple septa; cystidioles absent.................... gillesii A. David \& Rajchenb.

Generative hyphae only with clamp connections; fusoid cystidioles present

W. japonica

\section{Acknowledgements}

Special thanks are due to Prof. Leif Ryvarden (O. Norway), Drs. Bernard Rivoire (LY, France) and Pertti Salo (H, Finland) for loan of specimens. We express our gratitude to Prof. Yu-Cheng Dai (BJFC, China) for help in field collection. The research was supported by the Fundamental Research Funds for the Central Universities (No. BLYJ201403) and Beijing Higher Education Young Elite Teacher Project (No. YETP0774).

\section{References}

Chen, J.J. \& Cui, B.K. (2012) Studies on Wrightoporia from China 2. A new species and three new records from South China. Mycotaxon 21: 333-343.

http://dx.doi.org/10.5248/121.333

Chen, J.J. \& Cui, B.K. (2014) Phlebiporia bubalina gen. et. sp. nov. (Meruliaceae, Polyporales) from southwest China with a preliminary phylogeny based on rDNA sequences. Mycological Progress 13: 563-573.

http://dx.doi.org/s11557-013-0940-4 
Chen, J.J. \& Yu, H.Y. (2012) Studies on the genus of Wrightoporia from China 1. A new species described from Hunan Province, South China. Mycotaxon 120: 295-300.

http://dx.doi.org/10.5248/120.295

Corner, E.J.H. (1989) Ad polyporaceas 5. Beih Nova Hedwigia 96: 1-218.

Cui, B.K. (2013) Antrodia tropica sp. nov. from southern China inferred from morphological characters and molecular data. Mycological Progress 12: 223-230. http://dx.doi.org/10.1007/s11557-012-0829-7

Cui, B.K. \& Dai, Y.C. (2006) Wrightoporia (Basidiomycota, Aphyllophorales) in China. Nova Hedwigia 83: $159-166$. http://dx.doi.org/10.1127/0029-5035/2006/0083-0159

Cui, B.K. \& Dai, Y.C. (2013) Molecular phylogeny and morphology reveal a new species of Amyloporia (Basidiomycota) from China. Antonie van Leeuwenhoek 104: 817-827. http://dx.doi.org/10.1007/s10482-013-9994-1

Cui, B.K. \& Decock, C. (2013) Phellinus castanopsidis sp. nov. (Hymenochaetaceae) from southern China, with preliminary phylogeny based on rDNA sequences. Mycological Progress 12: 341-351. http://dx.doi.org/10.1007/s11557-012-0839-5

Cui, B.K., Zhao, C.L. \& Dai, Y.C. (2011) Melanoderma microcarpum gen. et sp. nov. (Basidiomycota) from China. Mycotaxon 116: 295-302. http://dx.doi.org/10.5248/116.295

Dai, Y.C. (2007) Amylosporus campbellii, a noteworthy polypore new to Southest Asia. Czech Mycology 59: 171-175.

Dai, Y.C. (2012) Polypore diversity in China with an annotated checklist of Chinese polypores. Mycoscience 53: 49-80. http://dx.doi.org/10.1007/s10267-011-0134-3

Dai, Y.C. \& Cui, B.K. (2006) Two new species of Wrightoporia (Basidiomycota, Aphyllophorales) from southern China. Mycotaxon 96: $199-206$

Dai, Y.C. \& Yuan, H.S. (2007) Type studies on polypores described by G. Y. Zheng and Z. S. Bi from southern China. Sydowia 59: $25-31$.

Dai, Y.C., Cui, B.K. \& Liu, X.Y. (2010) Bondarzewia podocarpi, a new and remarkable polypore from tropical China. Mycologia 102: $881-886$. http://dx.doi.org/10.3852/09-050

Dai, Y.C., Cui B.K., Yuan H.S., He S.H., Wei Y.L., Qin W.M., Zhou L.W. \& Li H.J. (2011) Wood-inhabiting fungi in southern China. 4. Polypores from Hainan Province. Annales Botanici Fennici 48: 219-231. http://dx.doi.org/10.5735/085.048.0302

David, A. \& Rajchenberg, M. (1987) A reevaluation of Wrightoporia and Amylonotus (Aphyllophorales, Polyporaceae). Canadian Journal of Botany 65: 202-209. http://dx.doi.org/10.1139/b87-027

Felsenstein, J. (1985) Confidence intervals on phylogenetics: an approach using bootstrap. Evolution 39: 783-791. http://dx.doi.org/10.2307/2408678

Hall, T.A. (1999) Bioedit: a user-friendly biological sequence alignment editor and analysis program for Windows 95/98/NT. Nucleic Acids Symposium Series 41: 95-98.

Hattori, T. (2003) Type studies of the polypores described by E.J.H Corner from Asia and West Pacific Areas 6. Species described in Tyromyces (3), Cristelloporia, Grifola, Hapalopilus, Heterobasidion, Ischnoderma, Loweporus, and Stecchericium. Mycoscience 44: 453-463. http://dx.doi.org/10.1007/s10267-003-0139-7

Hattori, T. (2008) Wrightoporia (Basidiomycota, Hericiales) species and their allies collected in Japan. Mycoscience 49: 56-65. http://dx.doi.org/10.1007/S10267-007-0389-X

He, S.H. \& Dai, Y.C. (2012) Taxonomy and phylogeny of Hymenochaete and allied genera of Hymenochaetaceae (Basidiomycota) in China. Fungal Diversity 56: 77-93. http://dx.doi.org/10.1007/s13225-012-0174-9

Jang, Y., Lee, S.W., Lim, Y.W., Lee, J.S., Hattori, T. \& Kim, J.J. (2013) The genus Wrightoporia in Korea. Mycotaxon 123: 335-341. http://dx.doi.org/10.5248/123.335

Larsson, E. \& Larsson, K.H. (2003) Phylogenetic relationships of russuloid basidiomycetes with emphasis on aphyllophoralean taxa. Mycologia 95: 1037-1065. http://dx.doi.org/10.2307/3761912

Larsson, K.H. (2007) Re-thinking the classification of corticioid fungi. Mycological research 111: 1040-1063. http://dx.doi.org/10.1016/j.mycres.2007.08.001 
Larsson, K.H., Larsson, E. \& Kõljalg, U. (2004) High phylogenetic diversity among corticioid homobasidiomycetes. Mycological research 108: 983-1002. http://dx.doi.org/10.1017/S0953756204000851

Li, H.J. \& Cui, B.K. (2013) Taxonomy and phylogeny of the genus Megasporoporia and its related genera. Mycologia 105: 368-383. http://dx.doi.org/10.3852/12-114

Li, H.J., Han, M.L. \& Cui, B.K. (2013) Two new Fomitopsis species from southern China based on morphological and molecular characters. Mycological Progress 12: 709-718. http://dx.doi.org/10.1007/s11557-012-0882-2

Lindblad, I. \& Ryvarden, L. (1999) Studies in neotropical polypores. 3. New and interesting Basidiomycetes (Poriales) from Costa Rica. Mycotaxon 71: 335-359.

Lowe, J.L. (1966) Polyporaceaea of North America. The genus Poria. State University College of Forestry at Syracuse University, New York.

Miller, S.L., Larsson, E., Larsson, K.H., Verbeken, A. \& Nuytinck, J. (2006) Perspectives in the new Russulales. Mycologia 98: 960970 http://dx.doi.org/10.3852/mycologia.98.6.960

Nylander, J.A.A. (2004) MrModeltest v2. Program distributed by the author. Evolutionary Biology Centre, Uppsala University.

Page, R.D.M. (1996) Treeview: application to display phylogenetic trees on personal computers. Computer Applications in the Biosciences 12: $357-358$.

Petersen, J.H. (1996) The Danish Mycological Society's colour-chart. Foreningen til Svampekundskabens Fremme, Greve, 6 pp.

Pouzar, Z. (1966) Studies in the taxonomy of the polypores 1. Česká Mykologie 20: 171-177.

Ronquist, F. \& Huelsenbeck, J.P. (2003) Mrbayes 3: Bayesian phylogenetic inference under mixed models. Bioinformatics 19: 1572 1574 . http://dx.doi.org/10.1093/bioinformatics/btg180

Ryvarden, L. (1982) Synopsis of the genus Wrightoporia. Nordic Journal of Botany 2: 145-149. http://dx.doi.org/10.1111/j.1756-1051.1982.tb01174.x

Ryvarden, L. (2000) Studies in neotropical polypores 7. Wrightoporia (Hericiaceae, Basidiomycetes) in tropical America. Karstenia 40: $153-158$.

Stalpers, J.A. (1996) The aphyllophoraceous fungi 2. Keys to the species of the Hericiales. Studies in Mycology 40: 37-185.

Swofford, D.L. (2002) PAUP*: Phylogenetic analysis using parsimony (*and other methods). Version 4.0b10. Sinauer Associates, Massachusetts.

Teixeira, A.R. (1992) New combinations and new names in the Polyporaceae. Revista Brasileira Botânica15: $125-127$.

Thompson, J.D., Gibson, T.J., Plewniak, F., Jeanmougin, F. \& Higgins, D.G. (1997) The Clustal_X windows interface: fexible strategies for multiple sequence alignment aided by quality analysis tools. Nucleic Acids Research 25: 4876-4882. http://dx.doi.org/10.1093/nar/25.24.4876

White, T.J., Bruns, T., Lee, S. \& Taylor, J. (1990) Amplification and direct sequencing of fungal ribosomal RNA genes for phylogenetics. In: Innis, M.A., Gelfand, D.H., Sninsky, J.J. \& White, T.J. (Eds.) PCR Protocols: A guide to methods and applications. Academic Press, San Diego, pp. 315-322.

Zhao, C.L. \& Cui, B.K. (2013) Morphological and molecular identification of four new resupinate species of Perenniporia (Polyporales) from southern China. Mycologia 105: 945-958. http://dx.doi.org/10.3852/12-201

Zhao, C.L., Cui, B.K. \& Dai, Y.C. (2013) New species and phylogeny of Perenniporia based on morphological and molecular characters. Fungal Diversity 58: 47-60. http://dx.doi.org/10.1007/s13225-012-0177-6

Zhou, L.W. \& Dai, Y.C. (2013) Taxonomy and phylogeny of wood-inhabiting hydnoid species in Russulales: two new genera, three new species and two new combinations. Mycologia 105: 636-649. http://dx.doi.org/10.3852/12-011 